\title{
Mechanisms of femtosecond laser- induced refractive index modification of poly(methyl methacrylate)
}

Article

Published Version

paper

Baum, A., Scully, P. J., Perrie, W., Liu, D. and Lucarini, V. (2010) Mechanisms of femtosecond laser-induced refractive index modification of poly(methyl methacrylate). Journal of the Optical Society of America B-Optical Physics, 27 (1). pp. 107111. ISSN 0030-3941 doi:

https://doi.org/10.1364/JOSAB.27.000107 Available at https://centaur.reading.ac.uk/16699/

It is advisable to refer to the publisher's version if you intend to cite from the work. See Guidance on citing.

To link to this article DOI: http://dx.doi.org/10.1364/JOSAB.27.000107

Publisher: The Optical Society of America

Publisher statement: This paper was published in Journal of the Optical Society of America B-Optical Physics and is made available as an electronic reprint with the permission of OSA. The paper can be found at the following URL on the OSA website: http://www.opticsinfobase.org/abstract.cfm?uri=josab-27-1-107.

Systematic or multiple reproduction or distribution to multiple locations via electronic or other means is prohibited and is subject to penalties under law.

All outputs in CentAUR are protected by Intellectual Property Rights law, including copyright law. Copyright and IPR is retained by the creators or other copyright holders. Terms and conditions for use of this material are defined in 
the End User Agreement.

www.reading.ac.uk/centaur

\section{CentAUR}

Central Archive at the University of Reading

Reading's research outputs online 


\title{
Mechanisms of femtosecond laser-induced refractive index modification of poly(methyl methacrylate)
}

\author{
Alexandra Baum, ${ }^{1}$ Patricia J. Scully, ${ }^{1, *}$ Walter Perrie, ${ }^{2}$ Dun Liu, ${ }^{2}$ and Valerio Lucarini ${ }^{3,4}$ \\ ${ }^{1}$ Photon Science Institute, The University of Manchester, Manchester M60 1QD, UK \\ ${ }^{2}$ Laser Group, Department of Engineering, University of Liverpool, Liverpool L69 3GQ, UK \\ ${ }^{3}$ Department of Mathematics, University of Reading, PO Box 220, Reading RG6 6AX, UK \\ ${ }^{4}$ Department of Meteorology, University of Reading, PO Box 243, Reading, RG6 6BB, UK \\ *Corresponding author: p.scully@manchester.ac.uk
}

Received September 24, 2009; accepted November 13, 2009; posted December 1, 2009 (Doc. ID 117467); published December 24, 2009

The mechanisms of refractive index change in poly(methyl methacrylate) by frequency doubled femtosecond laser pulses are investigated. It is demonstrated that positive refractive index modificaton can be caused by a combination of depolymerization and crosslinking. (C) 2009 Optical Society of America

OCIS codes: $350.3390,350.3450,160.5470$.

\section{INTRODUCTION}

The direct laser writing of refractive index modulated components, such as gratings and waveguides, for integrated photonic devices requires detailed understanding of the modification mechanism. Recent publications on the femtosecond laser irradiation of poly(methyl methacrylate) (PMMA) reported contradictory results for the sign of the refractive index change $\Delta n$. Zoubir et al. demonstrated negative $\Delta n$ surrounded by a compressed, tubular waveguide structure [1], while others observed positive $\Delta n$ due to densification in the focal volume [2-6]. The diverse results could be due to different writing regimes $(\mathrm{MHz}$ versus $\mathrm{kHz}$ repetition rate), temporal changes, or PMMA composition. Tubular waveguides with $\Delta n=2$ $\times 10^{-3}$ were produced using a high-repetition rate fs oscillator (25 MHz, $800 \mathrm{~nm}, 30 \mathrm{fs})$ [1]. The first symmetric waveguides in PMMA by beam shaped transversal writing ( $85 \mathrm{fs}, 800 \mathrm{~nm}, 1 \mathrm{kHz}, \Delta n=4.6 \times 10^{-4}$ ), were explained merely as chain scission causing volume contraction [4]. Temporary tubular waveguides developing into positive $\Delta n$ waveguides $(1 \mathrm{kHz}, 800 \mathrm{~nm}, 85 \mathrm{fs}$ laser) were explained by thermal expansion causing tensile stress to the surrounding area and subsequent volume contraction [5]. Multiphoton fs laser-inscribed positive $\Delta n$ gratings in PMMA (140 fs, $775 \mathrm{~nm}, 1 \mathrm{kHz}$ ) were compared to linearly absorbed ns pulses, in which the exposure dose determines a combination of crosslinking and degradation [3]. Heating to $70^{\circ} \mathrm{C}$ developed the modification $(1 \mathrm{kHz}$, $800 \mathrm{~nm}, 120 \mathrm{fs}$ ) from $\Delta n=0.6 \times 10^{-4}$ to $\Delta n=5.5 \times 10^{-4}$; chemical changes were not detected using Raman spectroscopy [6]. Previous results by the authors found depolymerization during fs laser modification of PMMA, together with increased refractive index of up to $\Delta n=4$ $\times 10^{-3}$ in the exposed regions [7]. Furthermore, temporal development of the modifications due to monomer diffusion was observed [8].
This work presents a range of analytical techniques to sample the modification mechanism in well-defined, pure grade PMMA to determine how the detected photochemical changes correspond to the observed $\Delta n$ and considers contributions of mechanisms, such as formation of absorption centers, molar refractivity of degradation products, monomer proportion within the polymer, stresses, and crosslink formation.

\section{DETECTION OF REFRACTIVE INDEX CHANGE MECHANISM}

The linear refractive index $n$ of a polymer is proportional to its density and depends on the chemical composition (including endgroups, additives, and impurities), molecular orientation, forces between the chains, and thermal history. Laser-induced refractive index changes are often explained as density changes. Some materials, such as fused silica, are densified by melting and rapid cooling as they are "frozen" at a fictive temperature [9]; conversely, most glassy materials, such as PMMA, undergo thermal expansion, and rapid cooling reduces density. A study of fs modification of high-purity PMMA by the authors indicated that $\Delta n$ was accompanied by a degradation process. Random chain scission by direct bond breaking caused broadening of the polymer molecular weight distribution (MWD), and an unzipping process led to monomer formation [7]. Depolymerization usually increases polymer chain volume as a result of the higher number of endgroups, causing expansion, or, when confined, internal material stresses. Stress-induced optical behavior of PMMA varies with material composition. Softeners, monomer, and impurities alter it and may even reverse its sign [10]. High-purity PMMA develops negative birefringence under stress [10], and depolymerization and expansion would reduce $n$. However, the measured $\Delta n$ was posi- 
tive, indicating additional mechanisms requiring alternative explanations to relate depolymerization to positive $\Delta n$. Both depolymerization and increased absorption at the UV absorption edge was shown after PMMA fs laser irradiation [7]. Similar effects in UV photodegradation studies were caused by unsaturated species [11,12]. Depolymerization increases polymer chain number and thus endgroups containing unsaturated bonds, creating both UV and IR spectral changes in PMMA. Embedded $\mathrm{C}=\mathrm{C}$ bonds exhibit electron transitions near $250 \mathrm{~nm}$ wavelength, affecting the absorption edge. Increased absorption related to induced $\Delta n$ was investigated by Kramers-Kronig (K-K) optical data inversion [13] of ultraviolet-visible (UV-VIS) absorption spectra. Degradation products of PMMA caused new absorption bands in the IR [11]. Thus, FTIR was investigated for the identification of chemical differences due to fs laser modification. After depolymerization the MWD is shifted, and the endgroups have a greater effect on the polymer properties. The influence of this on the theoretical molar refractivity of PMMA was tested by size exclusion chromatography (SEC). Volatile analysis (thermal desorption gas chromatography -mass spectrometry, TD GC-MS) during fs laser irradiation of PMMA detected photoreaction products. Polymer network strengthening factors, such as crosslinking or crystallinity, increase the thermal stability and decrease solubility of a polymer, since stronger forces between the chains cause densification. Therefore, thermal degradation and solubility of irradiated material were investigated by thermogravimetric analysis (TGA) and etching experiments.

\section{EXPERIMENTAL DETAIL}

Direct writing with an amplified Ti:sapphire fs laser (Clark-MXR CPA-2010; $1 \mathrm{kHz}$ repetition rate, $180 \mathrm{fs}$ pulse duration, frequency doubled $387 \mathrm{~nm}$ ) was employed. The laser beam (4 mm diameter) was focused by a $75 \mathrm{~mm}$ focal length lens below the surface of the sample, which was translated at $2 \mathrm{~mm} / \mathrm{s}$ to produce grating structures with a period of $\Lambda=10-20 \mu \mathrm{m}$. Pure PMMA was used for TD GC-MS, FTIR, and etching as solvent cast films (prepared as in [7]) and as bulk clinical grade Vistacryl CQ for UV-VIS spectroscopy and TGA. Gaseous reaction products were purged from the reaction cell into sorbent filled tubes and recovered with a Saturn 2200 GC-MS system. Compared with [7], the total split ratio was reduced to 3.1:1 for increased sensitivity, the temperature range was lowered to $40{ }^{\circ} \mathrm{C}$ up to $200{ }^{\circ} \mathrm{C}$ with a slower rate of $3.5^{\circ} \mathrm{C} / \mathrm{min}$ to include lower boiling point components and increase the separation, and the sampled mass-to-charge range was extended to $m / z=14-350$. Calibration using a directly injected monomer solution of known concentration enabled estimation of the amount of laser-generated MMA. SEC detected the MWD of modified regions dissolved in tetrahydrofuran (THF) [7]. A K-K relation was used to infer $\Delta n$ via absorbance measurements. The $\mathrm{K}-\mathrm{K}$ relations result from integral Hilbert transformations and are non-local operators; an absorption maximum is accompanied by decreased $n$ on its high-frequency side and increased $n$ on its low-frequency side. Thus, increased absorption at the UV-VIS edge after laser irradia- tion corresponds to positive $\Delta n$ in the visible spectrum. Transmission spectra were collected (Shimadzu 2401), and the wavelength-dependent extinction coefficient $\kappa$ was determined using Lambert-Beer's law: $\tau_{i}=I / I_{0}$ $=\exp [-(4 \pi / \lambda) \kappa C z]$. The internal transmittance $\tau_{i}$ (ratio of transmitted to the incident intensity) is attenuated depending on $\kappa$, the absorber concentration $C$, and the material thickness $z$. The $\mathrm{K}-\mathrm{K}$ algorithm was adapted from [13]. All samples were modified in a single scan $(N=1$; no overwrites to maximize $\Delta n$ ) because of the long processing time to create a sufficiently large area. Micro-FTIR spectra were collected in three replicates at different locations of unmodified and modified $\left(\Phi=0.446 \mathrm{~J} / \mathrm{cm}^{2}, \Lambda=20 \mu \mathrm{m}\right.$, $s=2 \mathrm{~mm} / \mathrm{s}, N=1$ ) PMMA film placed between two $\mathrm{CaF}$ disks (Hyperion Microscope with Bruker Equinox). The thermal stability was investigated using a 2950 Thermogravimetric Analyzer (TA Instruments), that ramped from $25-600{ }^{\circ} \mathrm{C}$ at $10 \mathrm{~K} / \mathrm{min}$ in $\mathrm{N}_{2}$. Chemical stability was tested by irrigation with $5 \%$ aqueous solution of methyl isobutyl ketone (MIBK) for $1 \mathrm{~min}$. The surface profile resulting from dissolved $\Delta n$ structures was measured using white light interference microscopy (Wyko NT1100).

\section{RESULTS AND DISCUSSION}

\section{A. Ultraviolet-Visible Absorption}

Figure 1 compares the transmittance of a set of lasermodified samples with unmodified PMMA, showing reduced transmittance at the absorption edge with increased laser exposure. At $\Phi=0.446 \mathrm{~J} / \mathrm{cm}^{2}$ weak ablation started. The corresponding difference extinction coefficients $\Delta \kappa=\kappa_{\text {modified }}-\kappa_{\text {unmodified }}$ are given in Fig. 2, left panel. By applying $\mathrm{K}-\mathrm{K}$ analysis to relative changes only, errors due to the truncated recorded spectrum are reduced and correction for surface reflection losses is unnecessary. For the geometry of the grating ( $20 \mu \mathrm{m}$ period, $10 \mu \mathrm{m}$ line width), a concentration $C=50 \%$ of modified to unmodified material was assumed. The $\mathrm{K}-\mathrm{K}$ inferred value of $\Delta n$ is shown in Fig. 2, right panel.

It is shown that increased absorption after laser irradiation can cause positive $\Delta n$, which increases with applied laser fluence. For a single scan sample near ablation threshold ( $\left.\Phi=0.446 \mathrm{~J} / \mathrm{cm}^{2}, \lambda=633 \mathrm{~nm}\right), \Delta n=1 \times 10^{-5}$ can be explained as due to increased absorption. This is below the detection limit of the direct measurement in [7],

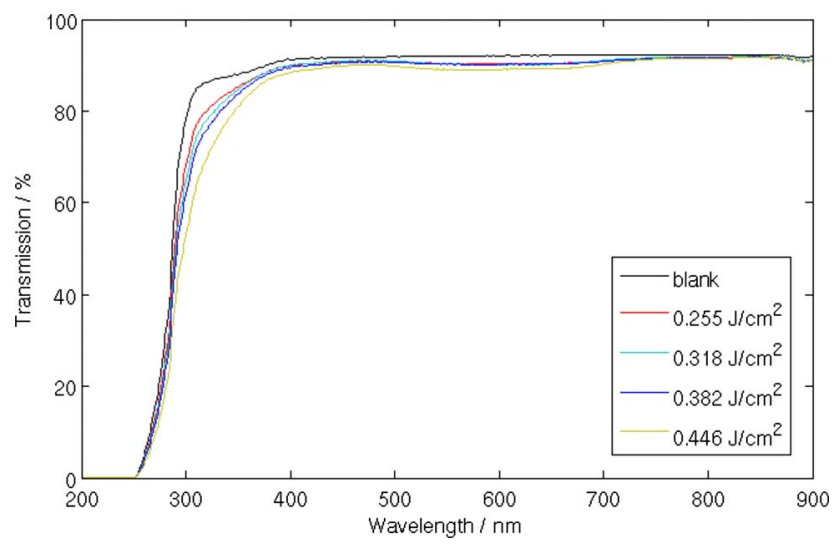

Fig. 1. (Color online) Transmittance of pure and laser-modified PMMA (1.1 mm Vistacryl CQ, $\Lambda=10 \mu \mathrm{m}, N=1$ ). 

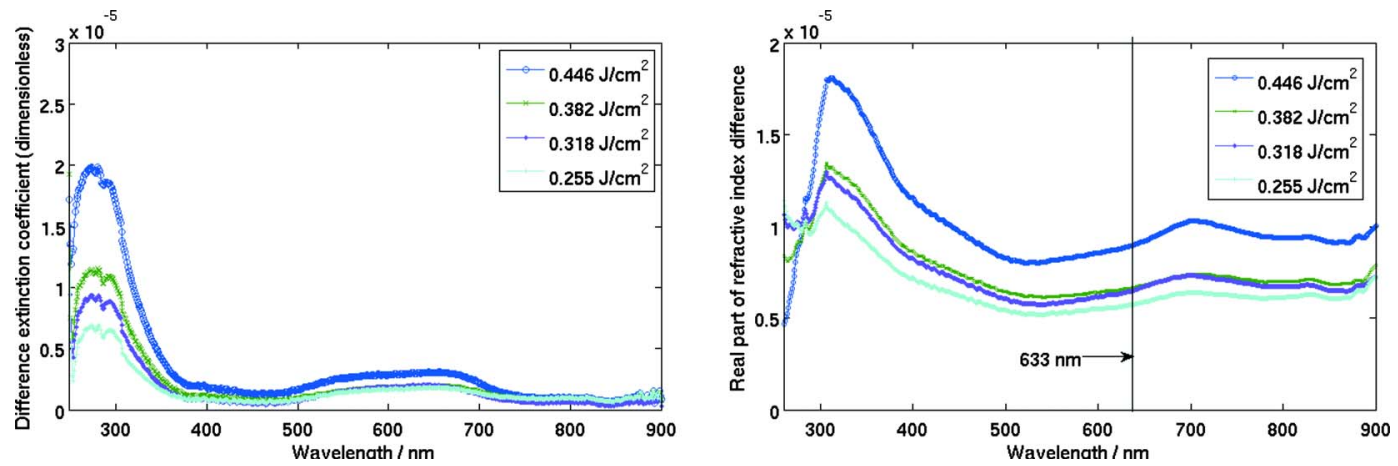

Fig. 2. (Color online) Difference extinction coefficient (left) and $\Delta n$ (right) between laser-modified and unmodified PMMA ( $\tau_{i}$ from Fig. $1, C=50 \%)$.

where a sample repeatedly exposed to low laser fluence was used. To better quantify the contribution of accumulated absorption centers to measured $\Delta n, \mathrm{~K}-\mathrm{K}$ analysis of samples with maximized change is required.

\section{B. Molar Refractivity}

The optical frequency Lorentz-Lorenz relation for transparent materials describes $n$ in terms of the molar refractivity $[R]$ and the material molar volume $V_{M}=M / \rho$ (repeat unit molecular weight over density) [14]:

$$
n=\left[\frac{1+2[R] / V_{M}}{1-[R] / V_{M}}\right]^{1 / 2} .
$$

The theoretical value of the molar refraction $[R]$ is the sum of all bond and group contributions of a molecule [14]). The group contributions for PMMA add up to $24.754 \mathrm{~cm}^{3}$, and assuming $V_{M}=86.5 \mathrm{~cm}^{3} / \mathrm{mol}$, the theoretical value for the refractive index at $\lambda=598 \mathrm{~nm}$ is $n_{D}$ $=1.484$, compared with the measured value of $n_{D}$ $=1.4900 \pm 0.0005$ at $20^{\circ} \mathrm{C}$ [14].

Depolymerization of PMMA causes new $\mathrm{C}_{5} \mathrm{H}_{7} \mathrm{O}_{2}$ chain ends containing an additional $\mathrm{C}=\mathrm{C}$ bond by a disproportion reaction. This structural modification alters $[R]$ and $V_{M}$ and, therefore, the index of the whole molecule. One proton is removed, so $M$ is reduced by $1 \mathrm{~g} / \mathrm{mol}$. The new double bond increases $[R]$ to $25.367 \mathrm{~cm}^{3}(\mathrm{C}=\mathrm{C}$ bond in main chain) or $25.409 \mathrm{~cm}^{3}(\mathrm{C}=\mathrm{C}$ bond of back bone with methyl side chain), respectively. Therefore, the refractive index of the molecule increases if the density remains constant.

The chain scission contribution to $\Delta n$ via structural modification was investigated using MWD data from SEC [7]. The total chain number was estimated by the integrated ratio of the SEC signal to the measured molecular weight, corresponding to monomer unit number divided by chain length. The chain end number is twice the chain number.

The mass fraction $v_{2}$ of chain ends within the polymer matrix is the chain end number normalized to sample concentration (integrated SEC signal). The abundance of chain ends increased with total laser fluence, e.g., $v_{2}$ $=3.51 \times 10^{-3}$ for repeatedly exposed PMMA $(\phi$ $=3.7 \mathrm{~J} / \mathrm{cm}^{2}, N=12$ ) compared to $v_{2}=2.68 \times 10^{-4}$ for unmodified PMMA. The effective $n$ of the two index component mixture was estimated (adapted from [15]) using the theoretical refractive indices $n_{1}=1.484$ for PMMA and $n_{2}=1.505$ for $\mathrm{C}=\mathrm{C}$ bond terminated PMMA:

$$
n=\left[\left(n_{2}^{2 / 3}-n_{1}^{2 / 3}\right) v_{2}+n_{1}^{2 / 3}\right]^{3 / 2},
$$

where $n_{1}$ is the index of the polymer matrix and $n_{2}$ of the second component, here representing the chain ends. The resulting index increase was calculated to be $\Delta n(\mathrm{C}=\mathrm{C})$ $=6.8 \times 10^{-5}$, confirming that depolymerization can theoretically raise $n$ through polymer chain end increase. Similar to the $\mathrm{K}-\mathrm{K}$ analysis of absorption centers, this method estimates the contribution of $\mathrm{C}=\mathrm{C}$ bonds. $\mathrm{Al}-$ though this is only a rough quantification, it indicates that the increased number of endgroups is unlikely to be the single source of the modification effect.

In addition, the effect of polymer side chain cleavage on $[R]$ was investigated. The structure of PMMA with the side chain replaced by a proton corresponds to polypropylene $\quad(\mathrm{PP}) \quad\left(\mathrm{C}_{3} \mathrm{H}_{6}\right) \mathrm{n} \quad\left([R]=13.909, \quad \rho=0.85 \mathrm{~g} / \mathrm{cm}^{3}, \quad M\right.$ $=42.08 \mathrm{~g} / \mathrm{mol}$ ) with theoretical $n=1.4738$. Cleaved PMMA side chains form methylformate $\mathrm{C}_{2} \mathrm{H}_{4} \mathrm{O}_{2}([R]=12.856, \rho$ $=0.98 \mathrm{~g} / \mathrm{cm}^{3}, \quad M=60.05 \mathrm{~g} / \mathrm{mol}$ ) with a theoretical $n$ $=1.3403$. Thus, both products of PMMA side chain scission would lower $n$ via $[R]$.

\section{Monomer Content and Fragmentation}

The refractive index of PMMA varies linearly between that of pure monomer $\left(n_{D}=1.414\right)$ and polymer $\left(n_{D}\right.$ $=1.492$ ), depending on the monomer content [10]. Structures with increased $\Delta n$ were demonstrated by photopolymerization of PMMA, containing residual monomer ( $\approx 10 \mathrm{wt} \%$ ) [16]. Clinical grade PMMA contains minimal residual monomer levels of $\leqslant 0.4 \mathrm{wt} \%$; thus polymerization cannot explain the observed increased $\Delta n$. Monomer formed during irradiation would counteract the observed increased $\Delta n$ when retained in the material but increase $n$ when diffusing out.

TD GC-MS enabled released MMA quantification during exposure. For single scans in PMMA with $1 \mathrm{~J} / \mathrm{cm}^{2}$, which is above the bulk damage threshold, a total of $>13.33 \mathrm{ng}$ MMA was detected over a 10 min sampling period. Sub-ablation threshold modification with $0.73 \mathrm{~J} / \mathrm{cm}^{2}$ released $>0.068 \mathrm{ng}$ MMA, indicating considerably increased monomer formation at the ablation threshold. The principal ions of fragments related to side chain cleavage for excimer laser degradation, such as methyl formate [17], were sought in the chromatogram; however, 


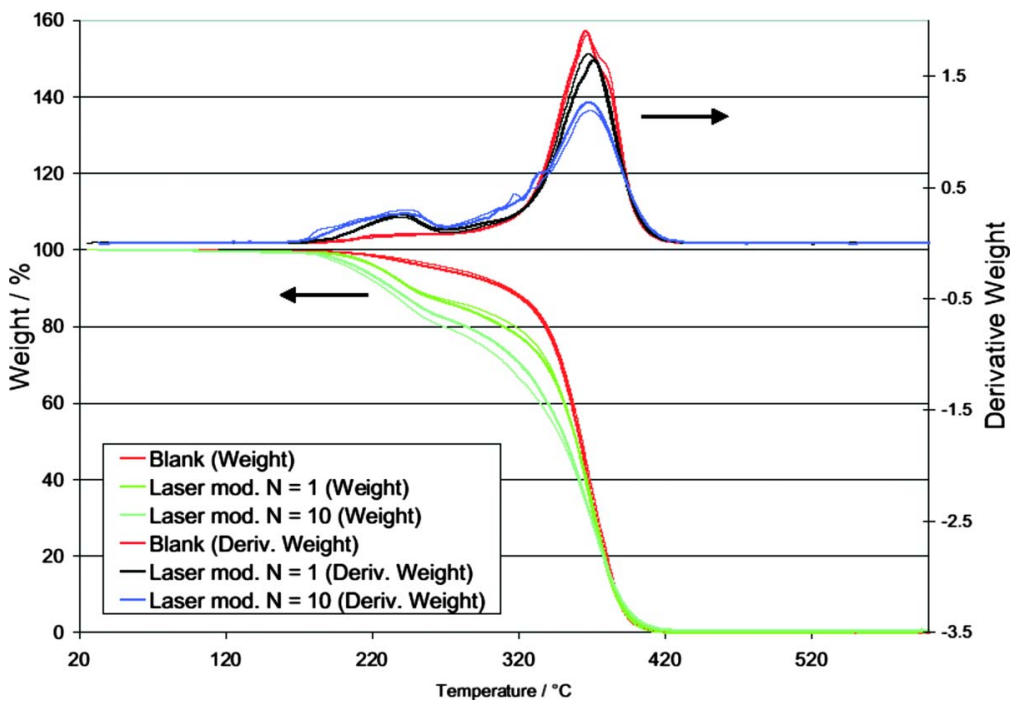

Fig. 3. (Color online) TGA of laser-modified and unmodified PMMA (260 $\mu \mathrm{m}$ initiator-free Vistacryl CQ; $\Phi=0.446 \mathrm{~J} / \mathrm{cm}^{2}, \Lambda=10 \mu \mathrm{m}$, $N=1,10)$.

photoproducts other than MMA were not found. This confirms direct cleavage of the polymer backbone initiated by random chain scission and propagated by unzipping to form monomer, as suggested in [7].

\section{Crosslinking}

The thermal degradation of a polymer reflects its composition and the polymerization process. For pure PMMA, thermal mass loss commences at $\approx 175{ }^{\circ} \mathrm{C}$ because of backbone degradation; at $\approx 225^{\circ} \mathrm{C}$, unsaturated endgroups degrade; and for temperatures $>300{ }^{\circ} \mathrm{C}$, random chain scission occurs [18]. The TGA of ns laser-deposited PMMA films demonstrated a higher decomposition temperature than the original material, attributed to crosslinking [19].

Figure 3 compares the thermal weight loss for pristine and fs laser-modified PMMA at $N=1$ and $N=10$ over scans, showing increased weight loss between 175 and $375^{\circ} \mathrm{C}$ with two additional peaks near 240 and $315^{\circ} \mathrm{C}$ after modification. The first peak is due to more unsaturated endgroups, increasing degradation below $250^{\circ} \mathrm{C}$. The second peak is formed by short polymer chains, which degrade rapidly by random scission. Between 375 and $425^{\circ} \mathrm{C}$, the decomposition rate of the modified material is slightly reduced, indicating more thermally stable crosslinked material. The maximum normalized weight difference for the blank and with $N=10$ laser-modified sample is about $0.4 \mathrm{wt} \%$ at $400{ }^{\circ} \mathrm{C}$ (the center tempera- ture related to crosslinking [18]). The contribution of partial crosslinking to $\Delta n$ was estimated with Eq. (2) assuming $n_{1}=1.4916$ for the unmodified and $n_{2}=1.5044$ for the totally crosslinked PMMA at $25^{\circ} \mathrm{C}$ [10]. For $v_{2}=0.4 \%$, $\Delta n=5 \times 10^{-5}$ was calculated, demonstrating that crosslinking, too, contributes to the index increase.

Figure 4 shows the surface relief grating revealed after etching with MIBK from a pre-etching intact surface. The laser-exposed material was removed, showing a profile of $\approx 70-80 \mathrm{~nm}$ depth and $\approx 5 \mu \mathrm{m}$ width, confirming formation of low MW compounds in the focal region for the $\mathrm{kHz}$ repetition rate fs laser modification of PMMA. Samples modified at the ablation threshold showed a shallow surface relief, with depth increasing from $<70 \mathrm{~nm}$ to $>1 \mu \mathrm{m}$ during etching. The etching experiments indicate that the laser-exposed PMMA consists mainly of degradation products with increased solubility. Indicators of crosslinking or other stability-increasing processes were not visible. However, partially crosslinked material, as detected by thermal degradation, can occupy localized areas, which are washed away together with the easily dissolved short chain fragment.

\section{E. Infrared Absorption}

The Fourier Transform Infrared Spectroscopy (FTIR) spectra of laser-modified and blank samples were almost identical, showing no evidence of photoproducts or dissolved monomer after the exposure, such as double bond
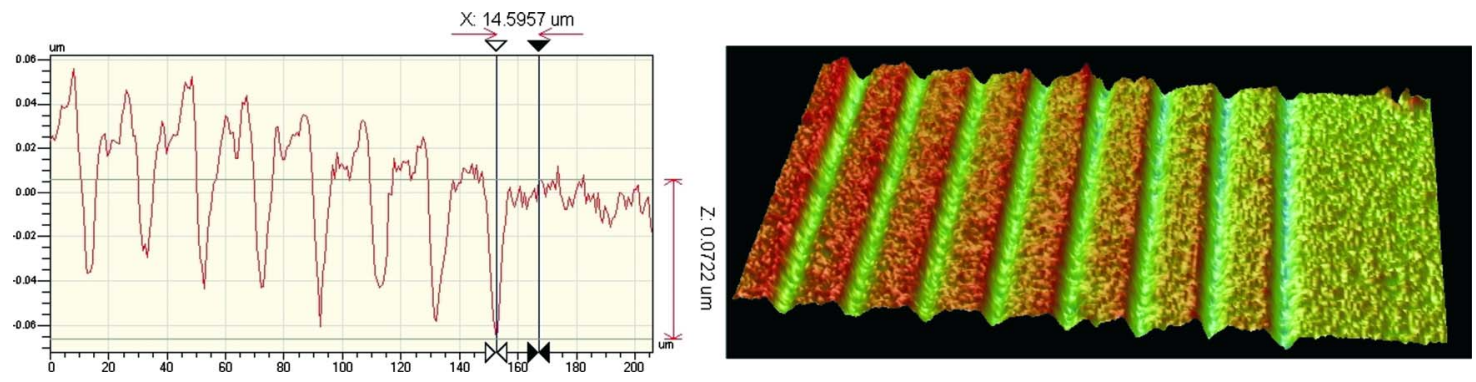

Fig. 4. (Color online) Surface profile of a previously sub-surface grating after etching in MIBK $\left(0.5 \mathrm{~mm}\right.$ Vistacryl CQ, $\Phi=0.446 \mathrm{~J} / \mathrm{cm}^{2}$, $\Lambda=20 \mu \mathrm{m}, N=1$ ). 
stretching around $1650 \mathrm{~cm}^{-1}$, reduced skeletal modes due to crosslinking at $1063 \mathrm{~cm}^{-1}$ [19], or shift of the $\mathrm{C}-\mathrm{C}-\mathrm{O}$ stretch at $1270 \mathrm{~cm}^{-1}$ by retained MMA [20]. Statistical analysis of the spectra by multivariate and discriminant function analysis identified the sample variance as noise, unrelated to the laser modification. This indicates that photoproducts have diffused out, and the chemical modification is below the FTIR detection limit, e.g., endgroup changes are overshadowed by unaffected polymer.

\section{CONCLUSION}

The optical data inversion of transmittance measurements, together with molecular weight analysis, thermal degradation, and etching experiments, gave new insights into the modification mechanism of $\mathrm{fs}$ laser-irradiated, pure PMMA. Positive $\Delta n$ was shown to be due to a combination of depolymerization and crosslinking. Increased UV absorption and reduced stability were detected. These are signs of depolymerization, related to lower molecular weight and a larger number of unsaturated endgroups. Using $\mathrm{K}-\mathrm{K}$ analysis, it was demonstrated that increased $n$ in the visible spectrum can be caused by enhanced absorption at shorter wavelengths. The presence of unsaturated endgroups was also shown to increase the theoretical $n$ of PMMA. These results explain how chain scission leads to positive $\Delta n$, which was to date unclear. In addition, thermally more stable material, attributed to crosslinking, was detected after laser irradiation of PMMA, further increasing the refractive index. The writing process by repeated irradiation, which was identified as the optimum method to create the largest modification [7], encourages depolymerization and the accumulation of absorption centers. The presented results indicate that both depolymerization and crosslinking contribute to the index change with similar magnitude $\left(\Delta n \approx 5 \times 10^{-5}\right.$ for each component). While their proportion is likely to be dose dependant, exact quantification remains difficult, and the presented summative $\Delta n$ magnitudes are currently unable to explain the total measured change of up to $\Delta n=4 \times 10^{-3}$ [7]. Therefore, future analyses of $\Delta n$-optimized samples with the presented methodology are planned.

\section{REFERENCES}

1. A. Zoubir, C. Lopez, M. Richardson, and K. Richardson, "Femtosecond laser fabrication of tubular waveguides in PMMA," Opt. Lett. 29, 1840-1842 (2004).

2. K. Ohta, M. Kamata, M. Obara, and N. Sawanobori, "Optical waveguide fabrication in new glasses and PMMA with temporally tailored ultrashort laser," Proc. SPIE 5340, 172-178 (2004).

3. C. Wochnowski, Y. Cheng, K. Meteva, K. Sugioka, K. Midorikawa, and S. Metev, "Femtosecond-laser induced formation of grating structures in planar polymer substrates," J. Opt. A, Pure Appl. Opt. 7, 493-501 (2005).

4. S. Sowa, W. Watanabe, T. Tamaki, J. Nishii, and K. Itoh, "Symmetric waveguides in PMMA fabricated by femtosecond laser pulses," Opt. Express 14, 291-297 (2006).

5. W. Watanabe, S. Sowa, T. Tamaki, K. Itoh, and J. Nishii, "Three-dimensional waveguides fabricated in PMMA by a femtosecond laser," Jpn. J. Appl. Phys. 45, L765-L767 (2006).

6. S. Hirono, M. Kasuya, K. Matsuda, Y. Ozeki, K. Itoh, H. Mochizuki, and W. Watanabe, "Increasing diffraction efficiency by heating phase gratings formed by femtosecond laser irradiation in polymethyl methacrylate," Appl. Phys. Lett. 94, 241122 (2009).

7. A. Baum, P. J. Scully, M. Basanta, C. L. P. Thomas, P. R. Fielden, N. J. Goddard, W. Perrie, and P. R. Chalker, "Photochemistry of refractive index structures in PMMA by femtosecond laser irradiation," Opt. Lett. 32, 190-192 (2007).

8. D. Liu, Laser Group, Department of Engineering, University of Liverpool, Liverpool L69 3GQ, UK; and Z. Kuang, W. Perrie, P. J. Scully, A. Baum, S. P. Edwardson, E. Fearon, G. Dearden, and K. G. Watkins are preparing a manuscript to be called "High speed ultrafast $3 \mathrm{D}$ refractive index micro-structuring of poly(methyl methacrylate)."

9. C. B. Schaffer, J. F. Garcia, and E. Mazur, "Bulk heating of transparent materials using a high-repetition-rate femtosecond laser," Appl. Phys. A: Solids Surf. 76, 351-354 (2003).

10. R. Vieweg and F. Esser, Kunststoff-Handbuch Band 4, Polymethacrylate-Herstellung, Verarbeitung und Anwendung (Carl Hanser, 1975).

11. S. Kuper and M. Stuke, "Ablation of uv-transparent materials with femtosecond uv excimer laser-pulses," Microelectron. Eng. 9, 475-480 (1989).

12. A. K. Baker and P. E. Dyer, "Refractive-index modification of PMMA thin-films by KrF-laser irradiation," Appl. Phys. A: Solids Surf. 57, 543-544 (1993).

13. V. Lucarini, J. J. Saarinen, K. E. Peiponen, and E. M. Vartiainen, Kramers-Kronig Relations in Optical Materials (Springer, 2005).

14. D. W. van Krevelen, Properties of Polymers: Their Estimation and Correlation with Chemical Structure, 2nd ed. (Elsevier, 1976).

15. H. Looyenga, "Dielectric constants of heterogeneous mixtures," Physica (Amsterdam) 31, 401-406 (1965).

16. J. Marotz, "Holographic storage in sensitized poly (methyl methacrylate) blocks," Appl. Phys. B: Photophys. Laser Chem. 37, 181-187 (1985).

17. G. B. Blanchet, P. Cotts, and C. R. Fincher, "Incubation: Subthreshold ablation of poly-(methyl methacrylate) and the nature of the decomposition pathways," J. Appl. Phys. 88, 2975-2978 (2000).

18. S. D. Smith, T. E. Long, and J. E. McGrath, "Thermogravimetric analysis of poly(alkyl methacrylates) and poly(methylmethacrylate-g-dimethyl siloxane) graft copolymers," J. Polym. Sci. A 32, 1747-1753 (1994).

19. E. Süske, T. Scharf, H.-U. Krebs, E. Panchenko, T. Junkers, M. Egorov, M. Buback, and H. Kijewski, "Tuning of crosslinking and mechanical properties of laser-deposited poly (methyl methacrylate) films," J. Appl. Phys. 97, 1-4 (2005).

20. H. Nagai, "Infrared spectra of stereoregular polymethyl methacrylate," J. Polym. Sci. 7, 1697-1714 (1963). 